\title{
Exploring the potential of UAV imagery for variable rate spraying in cotton defoliation application
}

\author{
Lili Yi ${ }^{1,2}$, Yubin Lan ${ }^{1,2}$, Hui Kong ${ }^{2}$, Fanxia Kong ${ }^{1,2}$, Huasheng Huang ${ }^{3}$, Xin $\operatorname{Han}^{1,2^{*}}$ \\ (1. National Sub-center for International Collaboration Research on Precision Agricultural Aviation Pesticide Spraying Technology, Zibo, \\ 255000, China; 2. College of Agricultural Engineering and Food Science, Shandong University of Technology, Zibo, 255000, China; \\ 3. College of Computer Sciences, Guangdong Polytechnic Normal University, Guangzhou 510665, China)
}

\begin{abstract}
Defoliants spraying is necessary to facilitate mechanized cotton harvesting. However, traditional uniform spraying usually decreases the defoliation effects and increases the costs. In order to address this problem, a variable rate spraying strategy was designed in the context of cotton defoliation. UAV multispectral imagery was collected in a cotton filed of Yellow River Basin in China, and the imagery was mosaicked into an ortho-photo. The index of NDVI and GNDVI were calculated, and the index maps were generated and later transformed into grid maps. A plant protection UAV was used for variable rate spraying, with the chemical dose proposed by the grid maps. Ground investigation before and after the spraying were conducted carefully to verify the defoliation effects. Investigation results showed that the proposed variable rate spraying strategy yielded satisfactory results with less defoliants, which demonstrated that this technology has potential in the cotton defoliation applications.
\end{abstract}

Keywords: multispectral remote sensing, cotton, defoliation, UAV

Citation: Yi L L, Lan Y B, Kong H, Kong F X, Huang H S, Han X. Exploring the potential of UAV imagery for variable rate spraying in cotton defoliation application. Int J Precis Agric Aviat, 2019; 2(1): 42-45.

\section{Introduction}

Defoliants spraying is a necessary step to facilitate cotton harvesting, and the use of defoliants has been proven to improve the defoliation quality and reduce the impurity content in cotton ${ }^{[1]}$. In nowadays, most farmers applied the defoliants uniformly throughout all the fields. However, the coverage of leaves varies across different sites, and uniform spraying would increase the costs while decrease the efficacy. In the sites with higher coverage of leaves, the spraying does is insufficient which decrease the defilation; in the sites with lower coverage of leaves, the defoliants dose is excessive which increase the costs. Variable rate spraying is an effective means to address this problem. This technology adjusts the chemical dose according to specific coverage of leaves at different sites, which may enhance the efficacy while reducing the use of defoliants ${ }^{[2]}$. In the process of variable rate spraying, a prescription map is required to provide decision support information ${ }^{[3]}$. Remote sensing offers an effective and efficient means for data collection, which can be used to generate the prescription map. In recent years, Unmanned Aerial Vehicle (UAV) has been widely used in crop monitoring ${ }^{[4-8]}$ as well as variable rate spraying applications ${ }^{[9,10]}$.

Castaldi et al. ${ }^{[9]}$ applied the UAV to collect multispectral imagery in the maize fields and processed the data into prescription map. Different herbicide strategies were applied according to the biomass data in the prescription map. Experimental results showed no difference in weed control between the patch spraying and uniform spraying, and the herbicide saving ranged from $14 \%$ to

Received date: 2019-10-19 Accepted date: 2019-10-22

Biographies: Lili Yi, Associate Professor of College of Agricultural Engineering and Food Science, Shandong University of Technology, P. R. China; Email: yili0001@sdut.edu.cn.

*Corresponding author: Xin Han, Associate Professor of College of Agricultural Engineering and Food Science, Shandong University of Technology, P. R. China. Email: hanxin@sdut.edu.cn.
39.2\%. López-Granados et al. ${ }^{[10]}$ collected high resolution UAV imagery, and used the OBIA method for weed mapping. Seven herbicide thresholds were designed according to the weed densities according to the weed cover maps. Experimental results showed that the OBIA method achieved high accuracy in weed recognition, and significant herbicide savings were observed in the two tested sunflower fields.

From the aforementioned related works, it can be seen that: (1) There is no literature currently available on variable rate spraying of cotton defoliants; (2) The analyzing methods (deep learning and OBIA methods) are somewhat complex, which is hard to be adopted by most farmers without professional backgrounds. The objective and contribution of this work is to: (1) explore the potential of variable rate technology on cotton defoliant spraying; (2) explore the potential of plant index for image processing, which is easy to generalize in real applications.

The rest of the paper is organized as follows. Section 2 describes the details of the data collection and image processing; section 3 describes the experimental results and discussion; section 4 gives the conclusion of this paper.

\section{Materials and Methods}

\subsection{Experimental site}

The test site is a cotton field located in Wudi County, Binzhou City in Shandong Province. The total area is more than 1.5 ha, and the soil of the test plot is saline and alkaline land. The One Film Three Rows Equal Spacing Cultivation Mode was adopted in the planting process. The equal row spacing of the cotton plants is $76 \mathrm{~cm}$, and the average plant height is $85 \mathrm{~cm}$. The tested cotton varieties are Jifeng 103 and Lumianyan 37. On the day of the first application, the temperature ranged from 22 to $26^{\circ} \mathrm{C}$, and the relative humidity was $43.4 \%$ to $54.5 \%$, with the wind speed from 0.40 to $1.15 \mathrm{~m} / \mathrm{s}$. On the day of the second application, the temperature of the second application was 20 to $25^{\circ} \mathrm{C}$, and the relative humidity was $47.4 \%$ to $62.5 \%$, with the wind speed from 


\subsection{1 to $1.23 \mathrm{~m} / \mathrm{s}$.}

\subsection{Data collection}

A six-rotor UAV with multispectral camera was used in data collection. The UAV was flying according to the planned routes. The forward lap and side lap were set to $90 \%$ and $80 \%$ respectively. The spatial resolution is $2.83 \mathrm{~cm}$, with a corresponding height of $30 \mathrm{~m}$. The multispectral camera consists of four bands: blue (475 nm center, $20 \mathrm{~nm}$ bandwidth), green (560 nm center, $20 \mathrm{~nm}$ bandwidth), red (668 nm center, $10 \mathrm{~nm}$ bandwidth), red edge (717 $\mathrm{nm}$ center, $10 \mathrm{~nm}$ bandwidth), and near-IR ( $840 \mathrm{~nm}$ center, 40 $\mathrm{nm}$ bandwidth). An illustration of planned route is shown as follows.

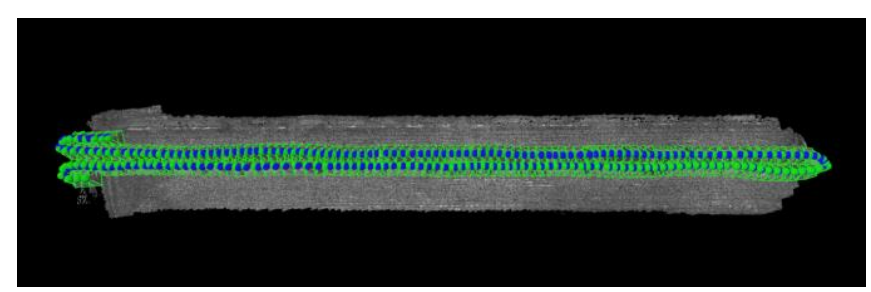

Figure 1 An illustration of the planned routes in data collection

\subsection{Data analyzing}

In order to reflect the coverage of the leaves, the Normalized Difference Vegetation Index (NDVI) and Green Normalized Difference Vegetation Index (GNDVI) are applied in this work. These indices were adopted because of their potential in biomass monitoring. Different from the disease monitoring and weed mapping, the coverage of cotton leaves are related with the biomass of the canopy. In this case, it is possible to use these indices to reflect the coverage of cotton, which can provide decision support information for the variable rate spraying.

Normalized Difference Vegetation Index (NDVI) is a popular index used to describe the crop grow conditions. The classical formula of NDVI is shown as follows,

$$
N D V I=\frac{N I R-R e d}{N I R+R e d}
$$

where, NIR stands for near-infrared region 750-900 nm. Since the NDVI indicates the growth of cotton, it can be used to guide the spraying of defoliant. The higher NDVI, the more leaves on the cotton canopy, which requires more defoliants. The variable rate spraying strategy in this work divided the field into several smaller areas, and applies one chemical dose for each sub area. In this case, the NDVI map was transformed into grid maps, which is showed in Figure 2. a. NDVI map

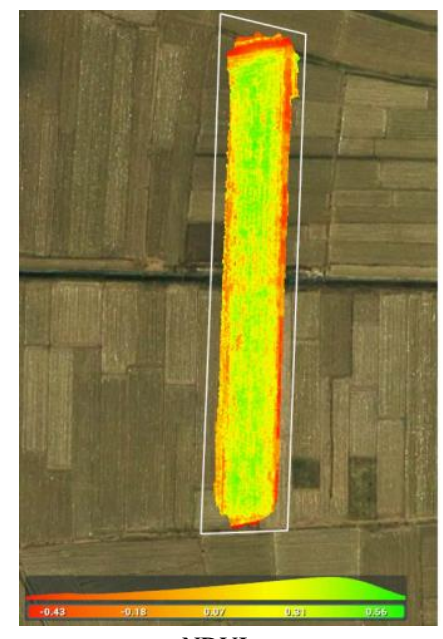

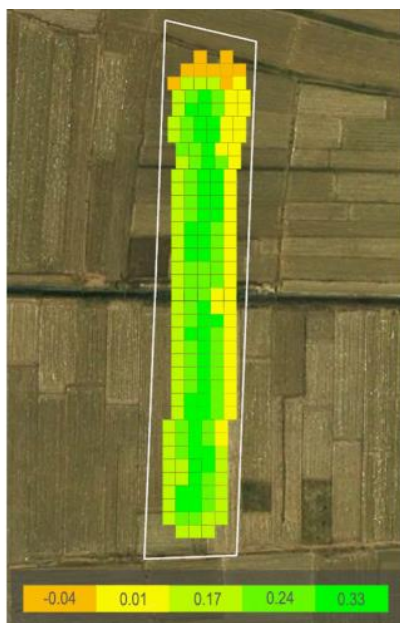

b. Grid map of NDVI
Figure 2 NDVI map and its grid map
Except NDVI, Green NDVI (GNDVI) is also a classical index which is frequently used to describe the crop growth condition. The definition of GNDVI is defined as follows,

$$
\text { GNDVI }=\frac{N I R-\text { Green }}{N I R+\text { Green }}
$$

The GNDVI was developed by [11] to map the global vegetation using EOSMODIS data. GNDVI is designed for chlorophyll estimation, which is potential in estimating the coverage of cotton leaves in this case. Similar with the NDVI, the GNDVI map was transformed into grid maps to support patch based spraying, which is showed in Figure 3.

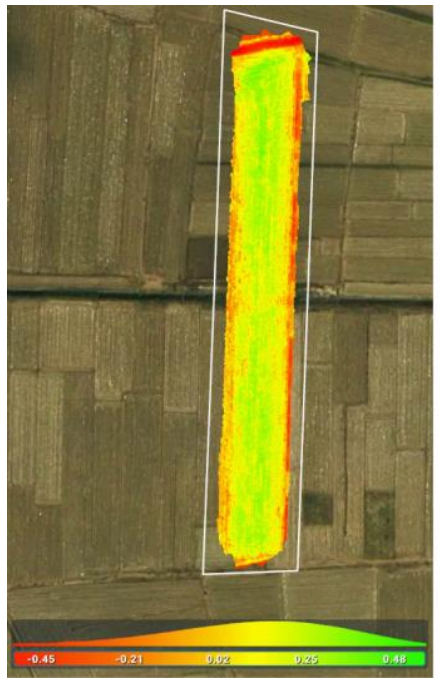

a. GNDVI map

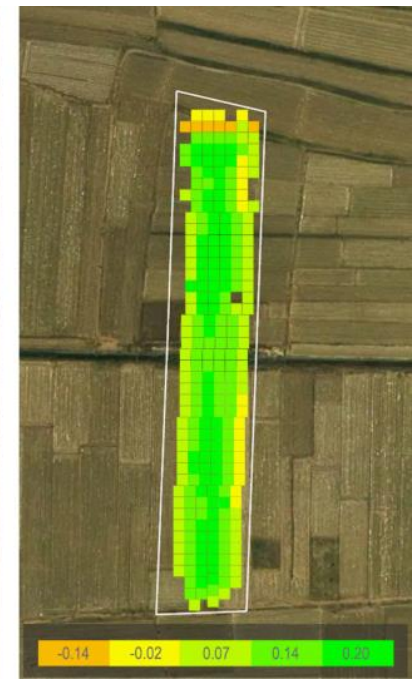

b. Grid map of GNDVI
Figure 3 GNDVI map and its grid map

\subsection{Variable rate spraying}

A four-rotor electric plant protection UAV (P30, XAG Co., Guangzhou, China) with a maximum tank capacity of $15 \mathrm{~L}$ was used in the experiment. This UAV supports autonomous obstacle avoidance and terrain awareness, with a working speed of 1-10 m/s. The spraying system consisted of a water pump, spray bar, control box, and nozzle. The nozzle adopted is a centrifugal nozzle, which drives the nozzle to rotate at a high speed through a motor, and breaks the liquid into fine droplet particles by centrifugal force. In order to ensure that the meteorological condition meets the operation requirement, the UAV is equipped with the sensors to detect the temperature, humidity and wind speed. The picture of the P30 UAV was showed in Figure 4.

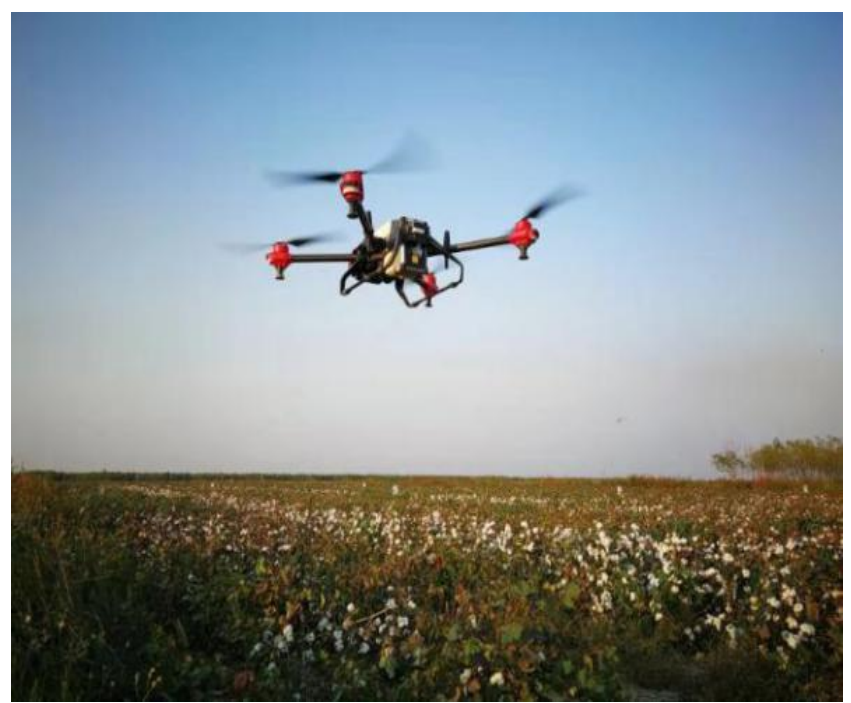

Figure 4 The plant protection UAV in the cotton fields 
The NDVI and GNDVI grid maps were considered to be the reference of the proposed dose. According to the data statistics, both grid maps reflected similar trends for all investigated sites, thus we decided to use only the GNDVI gird map for variable rate spraying. The quantitation of chemical dose based on GNDVI grid maps was showed in Table 1 . The chemical dose was selected under the instruction of agronomy experts.

Table 1 Spraying dose based on GNDVI grid maps

\begin{tabular}{cccc}
\hline Zone & $\begin{array}{c}\text { Average } \\
\text { value }\end{array}$ & $\begin{array}{c}\text { Area } \\
(\mathrm{Ha})\end{array}$ & Dose \\
\hline 1 & -0.14 & 0.06 & $40 \mathrm{~g}$ thidiazuron $+50 \mathrm{~mL}$ ethephon $+1 \mathrm{~mL}$ additives \\
2 & -0.02 & 0.38 & $40 \mathrm{~g}$ thidiazuron $+60 \mathrm{~mL}$ ethephon $+1 \mathrm{~mL}$ additives \\
3 & 0.07 & 0.26 & $40 \mathrm{~g}$ thidiazuron $+70 \mathrm{~mL}$ ethephon $+1 \mathrm{~mL}$ additives \\
4 & 0.14 & 0.38 & $40 \mathrm{~g}$ thidiazuron $+80 \mathrm{~mL}$ ethephon $+1 \mathrm{~mL}$ additives \\
5 & 0.20 & 0.51 & $40 \mathrm{~g}$ thidiazuron $+90 \mathrm{~mL}$ ethephon $+1 \mathrm{~mL}$ additives \\
Total & & 1.58 & \\
\hline
\end{tabular}

The cotton defoliants used 50\% active ingredient of thidiazuron and ethephon, and the additives are special adjuvants for flying defense produced by Anyang Quanfeng Biological Technology Co., Ltd.

The ground investigation before and after (5, 10, 15, 20 days later) the spraying tasks, the ground investigation were conducted to examine the chemical efficacy. In the ground investigation, the five-point sampling method was adopted in each investigation site. In each investigation site, four adjacent cotton were selected at each point, and the number of leaves of each cotton was recorded.

\section{Results and Discussion}

Traditional application uses the same dose for defoliants spraying. In our work, variable rate spraying was conducted to enhance the chemical effects and reduce the use of defoliants. In this work, the plant protection UAV of P30 (XAG, Co. Ltd., Guangzhou, China) was used to carry out variable spraying application, with the chemical dose proposed by the GNDVI grid maps. The operation height of the UAV was $2 \mathrm{~m}$, and the speed was $2 \mathrm{~m} / \mathrm{s}$, with the droplet size of 130 microns.

The number of cotton leaves before and after the spraying (5, 10, 15, 20 days later) was showed in Table 2. In table 2, A to E represent five different investigation sites, and 1 to 4 refers to four adjacent cotton in one site. From Table 2, it can be seen that: (1) the number of leaves decreases rapidly after the spraying, which demonstrated good defoliation effects; (2) the number of leaves over different cotton is approximate, which demonstrated a uniform efficacy over the whole field; (3) the defoliation effects are similar sites, which proved that the reduce of defoliants does not influence the chemical effects.

Table 2 Results of the cotton defoliant experiments

\begin{tabular}{|c|c|c|c|c|c|c|c|c|c|c|c|c|c|c|c|c|c|c|c|c|c|c|c|c|c|}
\hline & \multicolumn{5}{|c|}{ Before spraying } & \multicolumn{5}{|c|}{5 days later } & \multicolumn{5}{|c|}{10 days later } & \multicolumn{5}{|c|}{15 days later } & \multicolumn{5}{|c|}{20 days later } \\
\hline & A & B & $\mathrm{C}$ & $\mathrm{D}$ & $\mathrm{E}$ & A & B & $\mathrm{C}$ & $\mathrm{D}$ & $\mathrm{E}$ & A & B & $\mathrm{C}$ & $\mathrm{D}$ & $\mathrm{E}$ & A & B & $\mathrm{C}$ & $\mathrm{D}$ & $\mathrm{E}$ & A & B & $\mathrm{C}$ & $\mathrm{D}$ & $\mathrm{E}$ \\
\hline 1 & 191 & 198 & 199 & 206 & 199 & 130 & 129 & 133 & 149 & 131 & 89 & 85 & 92 & 99 & 87 & 37 & 31 & 38 & 29 & 26 & 18 & 10 & 16 & 15 & 17 \\
\hline 2 & 220 & 223 & 230 & 221 & 219 & 164 & 166 & 153 & 156 & 172 & 114 & 101 & 113 & 117 & 131 & 41 & 40 & 39 & 47 & 44 & 17 & 14 & 13 & 14 & 16 \\
\hline 3 & 261 & 259 & 266 & 275 & 236 & 193 & 186 & 197 & 205 & 170 & 100 & 96 & 109 & 110 & 99 & 45 & 49 & 51 & 50 & 45 & 19 & 20 & 25 & 25 & 20 \\
\hline 4 & 280 & 295 & 290 & 279 & 273 & 209 & 220 & 23 & 206 & 209 & 107 & 115 & 103 & 109 & 105 & 40 & 47 & 42 & 43 & 32 & 29 & 24 & 19 & 21 & 21 \\
\hline
\end{tabular}

Figure 5 shows the overview of the experimental site after spraying. The demonstrated field consists of treated area (in blue lines) and non-treated area (in red lines). It can be seen from Figure 5 that: (1) the defoliation effects in the treated area is much more significant than the non-treated area; (2) the efficacy of 15 days later is much significant than that of 5 days later, demonstrating that the defoliants may take some time for biological reaction on the cotton; (3) though different chemical dose was applied to different sites, all the sites present similar defoliation effects, demonstrating that the reduce of defoliants does not influence the efficacy.

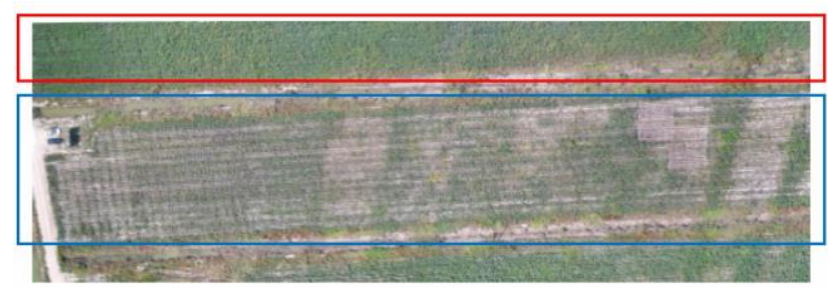

a. 5 days after defoliants spraying

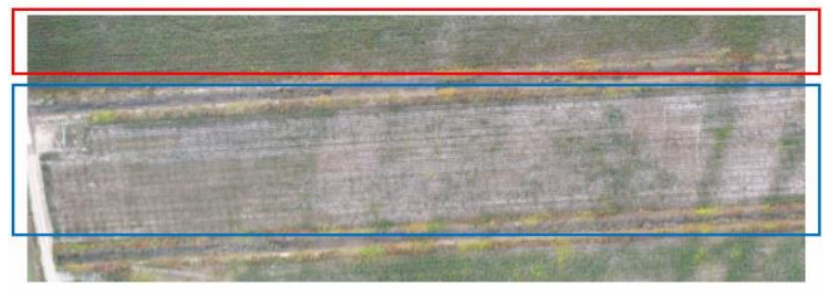

b. 15 days after defoliants spraying

Figure 5 The overview of the experimental site after the spraying

\section{Conclusions}

In this paper, a variable rate spraying experiment was conducted to improve the cotton defoliation while reducing the use of defoliants. The UAV was employed to collect high resolution imagery, and all the image patches were mosaicked into an orthophoto. The NDVI and GNDVI indices were calculated, which generated an index map. The index map was later transformed into grids, which may provide the sprayers with support information for each grid. A plant protection UAV was applied for variable rate spraying tasks, with the chemical dose proposed by the grid maps. After the spraying application, the efficacy investigation was conducted for continuous three weeks. The investigation results showed that the variable rate spraying strategy leads to good defoliation effects with less defoliants. Also, this work proved that a single plant index (GNDVI) can provide reliable support information for variable rate spraying, which will significantly simplify the workflow and make this technology easy to apply in real applications.

However, there are some shortage of this work. (1) To examine the feasibility of plant index (NDVI, GNDVI) for prescription map generation, more experiments need to be conducted for verification. (2) The chemical dose proposed by the prescription map should be transferred to the spraying UAV automatically. In this case, the flight control system and variable rate spraying system should be established. All these issues will be left as our future work. 


\section{Acknowledgements}

We acknowledge that this work was financially supported by Top Talents Program for One Case One Discussion of Shandong Province, Development Special Funds on Science and Technology to Guide Local by the Central Government, Program of Shandong Provincial Collaborative Innovation Center of Dry-farming Intelligent Agricultural Equipment, Key R \& D Program of Zibo (2019ZBXC053, 2019ZBXC143). We also thank the anonymous reviewers for their critical comments and suggestions to improve the manuscript.

\section{[References]}

[1] Xiao, Q., et al. Effect of aviation spray adjuvants on defoliant droplet deposition and cotton defoliation efficacy sprayed by unmanned aerial vehicles. Journal of Agronomy, 2019; 9: p. 217.

[2] Lan, Y., et al. Current status and future directions of precision aerial application for site-specific crop management in the USA. Computers and Electronics in Agriculture, 2010; 74: p. 34-38.

[3] Huang, H., et al. A fully convolutional network for weed mapping of unmanned aerial vehicle (UAV) imagery. PloS one, 2018; 13(4): p. e0196302.

[4] Pérez-Ortiz, M., et al. A semi-supervised system for weed mapping in sunflower crops using unmanned aerial vehicles and a crop row detection method. Applied Soft Computing, 2015; 37: p. 533-544.

[5] de Castro, A., et al. An Automatic random Forest-OBIA algorithm for early weed mapping between and within crop rows using UAV imagery. Remote Sensing, 2018; 10(3): p. 285

[6] Tamouridou, A. A., et al. Application of Multilayer Perceptron with Automatic Relevance Determination on Weed Mapping Using UAV Multispectral Imagery. Sensors (Basel, Switzerland), 2017; 17(10): p. 2307

[7] Albetis, J., et al. Detection of Flavescence dorée Grapevine Disease Using Unmanned Aerial Vehicle (UAV) Multispectral Imagery. Remote Sensing, 2017; 9(4): p. 308

[8] Alexandridis, T. K., et al. Novelty detection classifiers in weed mapping: silybum marianum detection on UAV multispectral images. Sensors (Basel, Switzerland), 2017; 17(9): p. 2007

[9] Castaldi, F., et al. Assessing the potential of images from unmanned aerial vehicles (UAV) to support herbicide patch spraying in maize. Precision Agriculture, 2017; 18(1): p. 76-94.

[10] López-Granados, F., et al. Early season weed mapping in sunflower using UAV technology: variability of herbicide treatment maps against weed thresholds. Precision Agriculture, 2016; 17(2): p. 183-199.

[11] Gitelson, A., Merzyak, M. N. and Lichtenthaler, H. K. Detection of red-edge position and chlorophyll content by reflectance measurements near $700 \mathrm{~nm}$. Journal of Plant Physiology, 1996; 148: 501-508. doi: 10.1016/S0176-1617(96)80285-9 